Article

\title{
Spatial Metrics of Interaction between CD163-Positive Macrophages and Cancer Cells and Progression-Free Survival in Chemo-Treated Breast Cancer
}

\author{
Brenton A. Maisel ${ }^{1}$, Misung Yi ${ }^{1}$, Amy R. Peck ${ }^{2}$, Yunguang Sun ${ }^{2}{ }^{\mathbb{D}}$, Jeffrey A. Hooke ${ }^{3}$, Albert J. Kovatich ${ }^{3}$, \\ Craig D. Shriver ${ }^{3}$, Hai Hu ${ }^{4}$, Marja T. Nevalainen ${ }^{2}$, Takemi Tanaka ${ }^{5}$, Nicole Simone ${ }^{6} \mathbb{C}$, Li Lily Wang ${ }^{7}$, \\ Hallgeir Rui ${ }^{2, *(\mathbb{B})}$ and Inna Chervoneva $1, *$ (i)
}

check for

updates

Citation: Maisel, B.A.; Yi, M.; Peck, A.R.; Sun, Y.; Hooke, J.A.; Kovatich,

A.J.; Shriver, C.D.; Hu, H.;

Nevalainen, M.T.; Tanaka, T.; et al. Spatial Metrics of Interaction between CD163-Positive Macrophages and Cancer Cells and Progression-Free Survival in Chemo-Treated Breast Cancer. Cancers 2022, 14, 308. https:// doi.org/10.3390/cancers14020308

Academic Editor: William Jacot

Received: 8 December 2021

Accepted: 5 January 2022

Published: 8 January 2022

Publisher's Note: MDPI stays neutral with regard to jurisdictional claims in published maps and institutional affiliations.

Copyright: (C) 2022 by the authors. Licensee MDPI, Basel, Switzerland. This article is an open access article distributed under the terms and conditions of the Creative Commons Attribution (CC BY) license (https:// creativecommons.org/licenses/by/ $4.0 /)$.
1 Department of Pharmacology and Experimental Therapeutics, Thomas Jefferson University, Philadelphia, PA 19107, USA; maiselbr@gmail.com (B.A.M.); Misung.Yi@jefferson.edu (M.Y.)

2 Department of Pathology, Medical College of Wisconsin, Milwaukee, WI 53226, USA; apeck@mcw.edu (A.R.P.); ysun@mcw.edu (Y.S.); mnevalainen@mcw.edu (M.T.N.)

3 John P. Murtha Cancer Center, Uniformed Services University and Walter Reed National Military Medical Center, Bethesda, MD 20814, USA; jhooke@hjfresearch.org (J.A.H.); akovatich@hjfresearch.org (A.J.K.); craig.d.shriver.civ@mail.mil (C.D.S.)

4 Chan Soon-Shiong Institute of Molecular Medicine at Windber, Windber, PA 15963, USA; H.Hu@wriwindber.org

5 Department of Pathology, University of Oklahoma Health Sciences Center, Stephenson Cancer Center, Oklahoma City, OK 73104, USA; Takemi-Tanaka@ouhsc.edu

6 Department of Radiation Oncology, Thomas Jefferson University, Philadelphia, PA 19107, USA; Nicole.Simone@jefferson.edu

7 Department of Translational Hematology and Oncology Research, Cleveland Clinic Foundation, 9500 Euclid Avenue, Cleveland, OH 44195, USA; WANGL9@ccf.org

* Correspondence: hrui@mcw.edu (H.R.); Inna.Chervoneva@jefferson.edu (I.C.)

Simple Summary: A majority of breast cancer deaths are caused by aggressive molecular subtypes that are at high risk of progression. Patients with high-risk breast cancer commonly receive first-line systemic chemotherapy. Chemotherapy exerts direct cytotoxic effects on proliferating cancer cells. In addition, significant effects of chemotherapy are mediated through immune-boosting anti-cancer mechanisms that counteract immunosuppressive tumor-associated macrophages (TAMs). The aim of our study was to evaluate the potential prognostic value of the prevalence and the spatial localization of $\mathrm{CD}_{163}{ }^{+} \mathrm{TAMs}$ in tumor tissue from breast cancer patients treated with chemotherapy after surgery. We developed a novel algorithm that identifies $\mathrm{CD} 163^{+}$TAMs in an objective manner and quantifies spatial interactions between $\mathrm{CD} 163^{+}$TAMs and cancer cells using distance-based metrics. Our results demonstrate that close spatial proximity of $\mathrm{CD} 163^{+}$TAMs to cancer cells and the average number of $\mathrm{CD}_{163}{ }^{+}$cells either directly adjacent to or within communicating distance of each cancer cell are independent predictors of unfavorable prognosis in breast cancer.

\begin{abstract}
Tumor-associated macrophages (TAMs) promote progression of breast cancer and other solid malignancies via immunosuppressive, pro-angiogenic and pro-metastatic effects. Tumorpromoting TAMs tend to express M2-like macrophage markers, including CD163. Histopathological assessments suggest that the density of CD163-positive TAMs within the tumor microenvironment is associated with reduced efficacy of chemotherapy and unfavorable prognosis. However, previous analyses have required research-oriented pathologists to visually enumerate CD163 ${ }^{+}$TAMs, which is both laborious and subjective and hampers clinical implementation. Objective, operator-independent image analysis methods to quantify TAM-associated information are needed. In addition, since M2-like TAMs exert local effects on cancer cells through direct juxtacrine cell-to-cell interactions, paracrine signaling, and metabolic factors, we hypothesized that spatial metrics of adjacency of M2-like TAMs to breast cancer cells will have further information value. Immunofluorescence histocytometry of $\mathrm{CD} 163^{+}$TAMs was performed retrospectively on tumor microarrays of 443 cases of invasive breast cancer from patients who subsequently received adjuvant chemotherapy. An objective and automated algorithm was developed to phenotype $\mathrm{CD} 163^{+}$TAMs and calculate their density
\end{abstract}


within the tumor stroma and derive several spatial metrics of interaction with cancer cells. Shorter progression-free survival was associated with a high density of $\mathrm{CD}_{163}{ }^{+}$TAMs, shorter median cancer-to-CD $163^{+}$nearest neighbor distance, and a high number of either directly adjacent CD163 ${ }^{+}$ TAMs (within juxtacrine proximity $<12 \mu \mathrm{m}$ to cancer cells) or communicating CD163 ${ }^{+}$TAMs (within paracrine communication distance $<250 \mu \mathrm{m}$ to cancer cells) after multivariable adjustment for clinical and pathological risk factors and correction for optimistic bias due to dichotomization.

Keywords: tumor-associated macrophages; cancer biomarkers; tumor immune microenvironment; spatially-resolved immunohistochemistry data; marked point patterns; nearest neighbor distance; microscopic image analysis; quantitative pathology; spatial interactions; breast cancer

\section{Introduction}

Despite improvements in screening, diagnosis, therapies, and surgery, breast cancer remains the second leading cause of cancer death in women [1]. The greatest reduction in breast cancer mortality would result from improved clinical management of patients diagnosed with high-risk tumors, such as those receiving chemotherapy as part of firstline treatment. While chemotherapy exerts direct cytotoxic effects on proliferating cancer cells, significant effects of chemotherapy are also mediated through immune-boosting anti-cancer mechanisms [2]. Conversely, emerging evidence from other solid tumor types indicates that strong intratumoral immunosuppressive mechanisms provide resistance to chemotherapy [3,4].

A plethora of research has demonstrated association of histological measures of antitumor immune activity with clinical outcome in multiple cancer types, with the main focus on tumor-infiltrating lymphocytes, particularly CD8+ cytotoxic T cells [5-13]. A comprehensive meta-analysis showed that the density of tumor-infiltrating lymphocytes is associated with favorable survival and predictive of pathologic complete response (pCR) in patients with HER2+ or triple-negative breast cancer (TNBC) subtypes after neoadjuvant chemotherapy [14]. In addition to the density of tumor-infiltrating lymphocytes [15], their differential spatial distribution as well as a spatial heterogeneity have prognostic value in gastrointestinal cancers [16-18], lung cancer [19-21], and breast cancer [22-28]. While cytotoxic $\mathrm{T}$ lymphocytes are tumor suppressive, tumor-associated macrophages (TAMs) exert immunosuppressive effects [29]. TAMs promote progression of breast cancer and other solid malignancies via local immunosuppressive, pro-angiogenic and pro-metastatic effects [30] that include enhancing cancer cell proliferation, invasion, and metastasis [31-33]. A high density of tumor-associated macrophages (TAMs) was significantly associated with poor survival in patients with breast cancer [14].

TAMs tend to display the M2-like macrophage phenotype and express CD163 [34]. While the pan-macrophage marker CD68 has been mostly used to identify TAMs in breast cancer tissues in earlier studies [35], more recent studies have established that a high density of $\mathrm{CD} 163^{+}$TAMs in breast tumors is associated with poor prognosis [36-39]. These studies each used visual counting of $\mathrm{CD}_{163}{ }^{+}$TAMs by pathologists but employed different methodologies to support an association of poor prognosis with elevated CD163 ${ }^{+}$TAMs in breast cancer. Medrek and colleagues relied on visual scoring of $\mathrm{CD}_{163^{+}}$cells in breast cancer into 4 ordinal categories ranging from 0 (none/absent) up to 3 (strong/dense) and found that infiltration of $\mathrm{CD}_{163}{ }^{+}$macrophages into tumor stroma, but not into tumor nests, had significant association with overall survival in a cohort of 144 patients [36]. Another study reported that high levels of CD163 ${ }^{+}$TAMs within the subjectively selected most TAM-enriched breast cancer regions were significantly associated with aggressive features, such as vessel invasion and non-luminal molecular subgroups and reduced survival in a cohort of 282 patients [39]. Jeong and colleagues found that a high number of $\mathrm{CD}_{163^{+}}$macrophages within tumor nests was an independent prognostic marker of reduced OS and DFS in 367 breast cancer patients [37]. Jamiyan and colleagues reported 
that infiltration of $\mathrm{CD} 163^{+}$TAMs, rather than CD68+, in both tumor stroma (TS) and tumor nests (TN) was associated with poor prognosis in patients with triple-negative breast cancer (TNBC) [38]. That study, similar to earlier reports [40,41], used methodology that involved initial identification of CD163 ${ }^{+}$TAM "hotspot" areas (five areas of TN and five areas of TS) with most abundant macrophages followed by counting the number of CD163-positive macrophages.

These initial investigations of $\mathrm{CD}_{163}{ }^{+}$TAMs in breast cancer have largely depended on research-focused pathologists to visually enumerate the cells, which is both laborious and subjective, and impedes implementation of these analyses into clinical practice. There is a great need for objective, machine-based approaches to effectively capture the information value that can be provided by the density and spatial proximity of CD163 ${ }^{+}$TAMs in breast cancer. Furthermore, none of the previously reported studies was focused on addressing the relationship between immunosuppressive CD163 ${ }^{+}$TAMs in patients with high-risk breast cancer who received chemotherapy.

We performed immunofluorescence-based histo-cytometry of CD163 ${ }^{+}$TAMs on tissue microarrays representing a retrospective cohort of 443 cases of invasive breast cancer from patients who subsequently received adjuvant chemotherapy. We developed objective, operator-independent image analysis methods to quantify the density of CD163 ${ }^{+}$TAMs and explored different spatial metrics of proximity to cancer cells as predictors of clinical outcome. To objectively determine the relationship between clinical outcome and proximity of $\mathrm{CD}_{163}{ }^{+} \mathrm{M} 2-$-like TAMs to breast cancer cells, three-color immunofluorescence (IF) images of breast cancer tissue microarrays were immunolabeled for cytokeratin (CK) and CD163 and counterstained with DAPI. Cell segmentation of digital images were performed by TissueStudio software (Definiens) followed by R-based procedures to provide spatial metrics as detailed in Methods and Figure 1.

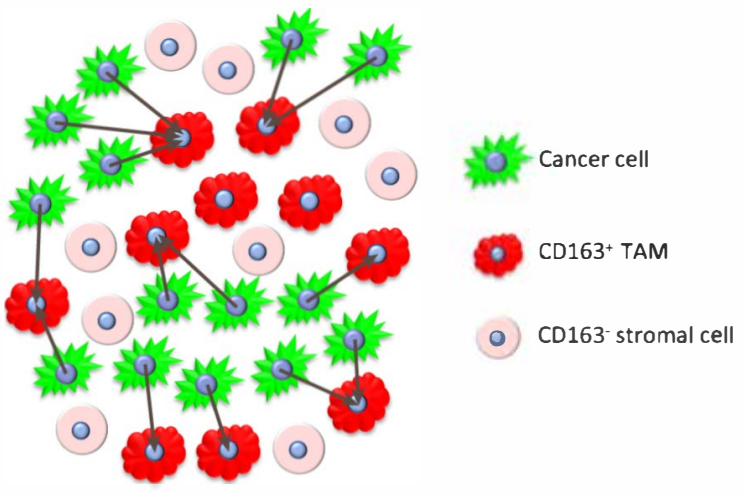

Figure 1. Computation of the nearest neighbor distances (NND) from each cancer cell to the nearest $\mathrm{CD}_{163}{ }^{+}$cell.

\section{Materials and Methods}

\subsection{Study Design}

This retrospective study analyzed archival specimens of primary invasive breast cancer from Thomas Jefferson University Hospital, Philadelphia, PA and the Clinical Breast Care Project (CBCP) tumor archive at the Walter Reed National Military Medical Center, Bethesda, MD. All specimen and data collections were conducted according to research protocols approved by local Institutional Review Boards. Archival formalin-fixed and paraffin-embedded specimens of primary invasive breast cancer tissue specimens from 1988-2010 were collected, reviewed by central pathologist (JAH), and representative cancer tissue regions were selected for inclusion in tissue microarrays (TMAs). TMAs were constructed using the Grandmaster tissue arrayer (3DHistech) with $0.6 \mathrm{~mm}$ core size. The specimens from unselected consecutive breast cancer cases were included into TMAs. The patients inclusion criteria for TMA were available tumor tissue and available clinical outcome data. Missing select clinico-pathological variable(s) was not an exclusion 
criterion, nor were there other inclusion or exclusion criteria. The study population was further limited to patients that (i) did not have distant metastases at the time of surgery; (ii) were treated with adjuvant chemotherapy; (iii) had CD163 stained IHC images that met the quality control. A small number of patients (9) had duplicated tissue cores. For these patients, the core with the higher number of $\mathrm{CD}_{163^{+}}$TAMs was used to compute CD163 $^{+}$ TAM-related metrics.

\subsection{Patient Cohort}

Patients received postoperative radiotherapy according to the treating physician's discretion. The progression-free survival (PFS) was defined as the time from diagnosis to the evidence of local, regional or distant recurrence. Only patients without distant metastatic disease at the time of diagnosis were included in the study cohort. The patients without progression were censored at the last follow-up time. Hormone receptor positivity was defined according to the definition in use at the time of diagnosis, which by immunohistochemistry ranged from $\geq 5 \%$ to $\geq 10 \%$ positivity during the study period of $1988-2010$. With respect to hormone treatment, patients with $\mathrm{HR}$-positive $(\mathrm{HR}+)$ tumors were classified as "non-compliant" if hormone treatment was recommended but patients did not receive this treatment.

\subsection{Immunohistochemistry}

Immunofluorescene immunohistochemistry (IF-IHC), slide scanning, and quantitative analysis of digitized images were performed in a manner blinded to outcome. Fluorescence immunohistochemistry for CD163 was performed on an autostainer (Agilent/Dako Omnis) using anti-CD163 antibody (Cat\# HPA046404, 1:4000; Sigma) followed by HRP-conjugated secondary antibody (Cat\# K4003, Agilent) and visualized using Cy5-tyramide as substrate (Cat\# NEL745001KT, Perkin Elmer), multiplexed with anti-pan-cytokeratin antibody (mouse monoclonal AE1/AE3 blend, Cat\# M3515, 1:100, Agilent) with Alexa-Fluor-555labeled secondary antibody (Cat\# A21422, Thermo Fisher) to identify cancer cells, followed by counterstaining with DAPI to visualize cell nuclei as previously described [42-44]. Stained slides were scanned at $20 \times$ magnification on the Pannoramic Flash 250 scanner (3DHistech) and fluorescent images were captured in three channels (Cy5, Cy3/Alexa-555 and DAPI). Cell segmentation of digital images was performed by TissueStudio software (Definiens) and CD163 immunoreactivity was computed for individual cancer cells and stromal cells for each tumor core. The operators were blinded to patient IDs and clinical outcomes. Some tissue cores were excluded from further analysis if the quality of the staining was deemed substandard or if less than 10 cancer cells were identified.

\subsection{Identification of $\mathrm{CD} 163^{+}$TAMs}

The image data included spatial localization information ( $\mathrm{x}-\mathrm{y}$ coordinates of cell centroids) for cancer and cells within the tumor stroma. The CD163-signal in cancer cells in each tissue core were viewed as background noise and were used to compute the $99 \%$ confidence $95 \%$ content non-parametric upper tolerance limit (UTL) for background CD163 signals. The quantile cutoffs for UTLs were selected using Table A.16 in [45] with the smallest sample size of 10 and using linear interpolation for the sample sizes not included in this table. Cell within tumor stroma were identified as CD163 ${ }^{+}$TAMs if their CD163 expression levels were above the background UTL for the corresponding tissue core. The identification procedure for CD163 ${ }^{+}$TAMs identification was implemented in Python [46].

\subsection{Spatial Metrics of Interaction between $C D 163^{+}$TAMs and Cancer Cells}

The spatial localization of cells was jointly considered for cancer cells, CD163 ${ }^{+}$TAMs, and CD163- stromal cells, and viewed as a multitype marked point pattern (MMPP) [47]. The resulting MMPPs were used to compute the spatial interaction metrics based on the distributions of the nearest neighbor distances (NND) between individual cancer cells and the nearest $\mathrm{CD}_{163^{+}} \mathrm{TAM}^{\mathrm{T}}$ and $\mathrm{CD} 163^{+}$TAM density metrics. Similar to visual enumeration 
of $\mathrm{CD} 163^{+}$TAMs in a region of interest by a pathologist, we considered the total count of $\mathrm{CD}_{163}{ }^{+}$TAMs within the sampled area of each tumor tissue core $\left(0.28 \mathrm{~mm}^{2}\right)$. The proportion of $\mathrm{CD}_{163}{ }^{+}$TAMs within the stromal compartment of each tumor tissue core was also computed. Neither of these traditional metrics needs information on the spatial localization of cancer cells and immune cells. The spatial NND-based metrics included summary statistics of the NND distributions for each tissue core: (i) 10th percentile, (ii) 25th percentile (i.e. 1st quartile), and (iii) the median (50th percentile). Figure 1 illustrates the computation of the NNDs from cancer cells to their nearest CD163 ${ }^{+}$TAM (grey arrows). In addition, we considered the average counts of adjacent CD163 ${ }^{+}$TAMs to each cancer cell (within $12 \mu \mathrm{m}$ distance of direct cell-to-cell or juxtacrine signaling) and communicating $\mathrm{CD} 63^{+}$TAMs to each cancer cell (within $250 \mu \mathrm{m}$ cyto/chemokine communication or paracrine signaling distance). The adjacent distance of $12 \mu \mathrm{m}$ is approximately equal to the estimated median diameter of cancer cells. The choice of $250 \mu \mathrm{m}$ is based on results of Francis and Palsson (1997) who estimated that $250 \mu \mathrm{m}$ is the maximum tissue distance over which a single cell can effectively communicate via paracrine cyto/chemokine signaling) [48]. All spatial metrics were computed using the R package 'spatstat' [49]. The tumor cores that had zero count of CD163 ${ }^{+}$TAMs (67 of the analyzed 443) were included in all analyses with the counts-based CD163 metrics equaled to zero and the NND-based metrics truncated at $600 \mu \mathrm{m}$, which is the diameter of the TMA tissue core.

\subsection{Dichotomization of CD163 Metrics and Association with Clinicopathologic Characteristics and Progression-Free Survival}

The correlation between CD163 metrics was evaluated using the Spearman correlation coefficient. The association between CD163 metrics and categorical clinicopathological factors was evaluated using the Kruskal-Wallis test. The optimal cutoff for each CD163 metrics with the corresponding $90 \%$ confidence interval was determined using the bootstrap approach. First, 1000 bootstrap samples were drawn with replacement from the study cohort. In each bootstrap sample, the survival tree model with 10-fold cross-validation ( $R$ package rpart [50]) was used to establish an objective data-driven optimal cutoff for dichotomizing the CD163 metric. The median of the resulting sample of 1000 cutoffs was used as the optimal cutoff for each CD163 metrics, and the corresponding non-parametric $90 \%$ confidence limits are 5th and 95th percentiles of the distribution of 1000 bootstrapbased cutoffs. The association between dichotomized CD163 metrics and categorical clinicopathological factors was evaluated using the Fisher's exact test or its extension for more than 2 categories. For the continuous age variable, the two-sample Wilcoxon SignedRank test was used. The $p$-values for testing associations between multiple continuous or dichotomized CD163 metrics and clinicopathological factors were adjusted for multiple testing using Holm's method which controls family-wise error rate [51].

Each CD163 metric computed was evaluated as a dichotomized predictor of PFS in a univariate Cox model and multivariable Cox model that included standard clinicopathological prognostic factors of PFS: age, race (white vs. non-white), histologic grade, node status, tumor size $(<2 \mathrm{~cm}, 2-5 \mathrm{~cm},>5 \mathrm{~cm})$, radiation therapy, chemotherapy, and hormone therapy non-compliance (vs. the compliant reference category). The parsimonious model was selected a priori including clinicopathological prognostic factors but not the CD163 markers. The proportional hazards assumption was evaluated for all covariates in the Cox models and covariates that violated the proportional hazard assumptions were incorporated in the models as strata variables.

\subsection{Internal Validation of Dichotomized CD163 Metrics}

The internal validation of dichotomized $\mathrm{CD}_{163^{+}}$metrics was performed using the bootstrap optimism correction procedure [52]. Multiple imputation was used for missing values for clinicopathologic covariates for some patients $(0.6 \%$ to $10 \%$ for all clinicopathological covariates, but higher level for hormone compliance, Table 1, Supplementary Tables S2-S7). Forty (40) imputed datasets were created using the multivariate imputation 
by chained equations (MICE) algorithm. For each covariate, missing values were imputed by univariate models for corresponding outcome type using the fully conditional specification [53]. The bootstrap optimism correction algorithm was applied to each imputed data set. Then results for all imputed data sets were averaged using Rubin's rule [54]. The following steps of the bootstrap optimism correction algorithm were performed for each imputed data set. First, 500 bootstrap samples were drawn with replacement from each imputed data set with all patients. In each bootstrap sample, the survival tree model with 10-fold cross-validation ( $\mathrm{R}$ package rpart) was used to establish an objective data-driven optimal cutpoint for dichotomizing the CD163 metric. The cutpoint from the current bootstrap sample was used to compute the log hazard ratio for the univariate and multivariate Cox models in the current bootstrap sample ("Bootstrap performance") and in the main sample ("Test performance"), and the optimism in hazard ratio estimation was computed as the difference between log hazard ratio for "Bootstrap performance" and for "Test performance". The mean optimism estimate was computed as the average optimism over 500 bootstrap samples. The cutpoint for dichotomizing individual spatial CD163 metrics was also established in the imputed data set and its "apparent performance" was computed as the log hazard ratio for dichotomized CD163 metric in the multivariable Cox models. Finally, the optimism-corrected performance estimates were computed by subtracting the mean optimism estimates from the apparent performance estimates [52]. The optimal cutpoints based on bootstrap samples were used to compute bootstrap-based non-parametric $90 \%$ confidence intervals for dichotomization cutpoints. The outcome and association analyses were performed using $R$ [55].

Table 1. Associations between dichotomized count of CD163+ cells and other prognostic factors.

\begin{tabular}{|c|c|c|c|c|c|c|}
\hline & \multirow[b]{3}{*}{ Total N } & \multicolumn{4}{|c|}{ Number of CD163+ Cells per $0.28 \mathrm{~mm}^{2}$} & \multirow[b]{3}{*}{$p(\mathcal{E})$} \\
\hline & & \multicolumn{3}{|c|}{ Low } & \multirow{2}{*}{$\begin{array}{c}\text { High } \\
\%\end{array}$} & \\
\hline & & $\mathbf{N}$ & $\%$ & $\mathbf{N}$ & & \\
\hline & 443 & 377 & $85 \%$ & 66 & $15 \%$ & \\
\hline HR- & 93 & 62 & $67 \%$ & 31 & $33 \%$ & $<0.001$ \\
\hline $\mathrm{HR}+$ & 349 & 314 & $90 \%$ & 35 & $10 \%$ & \\
\hline Missing & 1 & 1 & $100 \%$ & 0 & $0 \%$ & \\
\hline White & 101 & 81 & & 20 & $20 \%$ & 1.000 \\
\hline Non-White & 333 & 288 & $86 \%$ & 45 & $14 \%$ & \\
\hline Unknown & 9 & 8 & $89 \%$ & 1 & $11 \%$ & \\
\hline Histological Grade 1 & 119 & 109 & $92 \%$ & 10 & $8 \%$ & 0.018 \\
\hline Histological Grade 2 & 177 & 157 & $89 \%$ & 20 & $11 \%$ & \\
\hline Histological Grade 3 & 145 & 109 & $75 \%$ & 36 & $25 \%$ & \\
\hline Missing & 2 & 2 & $100 \%$ & 0 & $0 \%$ & \\
\hline Tumor Size <2 cm & 214 & 191 & $89 \%$ & 23 & $11 \%$ & 1.000 \\
\hline Tumor Size $2-5 \mathrm{~cm}$ & 171 & 136 & $80 \%$ & 35 & $20 \%$ & \\
\hline Tumor Size $>5 \mathrm{~cm}$ & 58 & 50 & $86 \%$ & 8 & $14 \%$ & \\
\hline Node Negative & 211 & 179 & $85 \%$ & 32 & $15 \%$ & 1.000 \\
\hline Node Positive & 223 & 190 & $85 \%$ & 33 & $15 \%$ & \\
\hline Missing & 9 & 8 & $89 \%$ & 1 & $11 \%$ & \\
\hline Her2 Negative & 375 & 321 & $86 \%$ & 54 & $14 \%$ & 1.000 \\
\hline Her2 Positive & 54 & 44 & $81 \%$ & 10 & $19 \%$ & \\
\hline Missing & 14 & 12 & $86 \%$ & 2 & $14 \%$ & \\
\hline Radiation $=$ Yes & 255 & 219 & $86 \%$ & 36 & $14 \%$ & 1.000 \\
\hline Radiation $=$ No & 188 & 158 & $84 \%$ & 30 & $16 \%$ & \\
\hline Horm Tx Non-Compliant & 98 & 89 & $91 \%$ & 9 & $9 \%$ & 0.003 \\
\hline Horm Tx Compliant & 45 & 40 & $89 \%$ & 5 & $11 \%$ & \\
\hline Unknown & 207 & 186 & $90 \%$ & 21 & $10 \%$ & \\
\hline
\end{tabular}

(E) adjusted for multiple testing using Holm's method. 


\section{Results}

The study cohort included 443 cases of locoregional HR+ and HR-breast cancer treated with adjuvant chemotherapy for whom tissue sample included at least 10 cancer cells. The clinical follow-up ranged from 1 month to 238 months with a median follow-up time of 64 months. There were 77 progressions in the study cohort of 443 patients (17.4\%). Table 1 details the patient characteristics and their association with the dichotomized standard metric of $\mathrm{CD}_{163}{ }^{+}$TAM count per tissue core.

Examples of breast cancer tissue core IF-IHC images are shown in Figure 2A-C with the corresponding multitype marked point patterns (MMPPs) of cancer cells (green), $\mathrm{CD} 63^{+}$TAMs (red), and CD163 ${ }^{-}$stromal cells (gray; Figure 2D-F), and the corresponding distributions of nearest neighbor distances (NNDs) from cancer cells to the nearest CD163 ${ }^{+}$ TAM with metrics of 10th percentile, 25th percentile (lower quartile Q1) and median NNDs (Figure 2G-I).

For the tumor core presented in Figure 2B, the NND distribution from cancer cells to $\mathrm{CD} 163^{+}$TAMs (Figure $2 \mathrm{H}$ ) is reflective of greater proximity (smaller range and smaller values of all percentiles, including 10th, 25th and median) than the NND distributions in Figure 2G,I, which corresponds to the tumor cores in Figure 2A,C. Such more proximal distribution corresponds to a higher prevalence of CD163 ${ }^{+}$TAMs found next to cancer cells in MMPP (Figure 2E). In contrast, the majority of CD163 ${ }^{+}$TAMs in Figures 2D,F are more separated of from cancer cells and the corresponding NND distributions in Figures 2G,I are wider and have larger values of all percentiles, including the 10th and 25th percentile and the median. As described in the Methods section, we also computed alternative spatial proximity metrics of the average number of $\mathrm{CD}_{163^{+}}$TAMs that are (i) directly adjacent to (within $12 \mu \mathrm{m}$ distance or (ii) within paracrine cyto/chemokine communicating distance (within $250 \mu \mathrm{m}$ distance) [48] of each cancer cell.

We first examined the correlations among all CD163 ${ }^{+}$TAM-related spatial metrics, including count and proportion of CD163 ${ }^{+}$TAMs, cancer-to-CD163 ${ }^{+}$NND-based metrics, and metrics of $\mathrm{CD}_{163^{+}}{ }^{\mathrm{TAM}}$ proximity to cancer cells (average numbers of adjacent or communicating CD163 ${ }^{+}$TAMs) (Supplementary Figure S1 and Supplementary Table S1). As expected, the traditional count of CD163 ${ }^{+}$TAMs in the tumor core and the proportion of CD163 ${ }^{+}$TAMs among stromal cells were highly correlated (coefficient $=0.74 ; 95 \% \mathrm{CI}$ : $0.69 ; 0.78)$. The average number of communicating CD163 ${ }^{+}$TAMs was highly correlated with CD163 ${ }^{+}$TAM count (coefficient $=0.989 ; 95 \%$ CI: $0.987 ; 0.991$ ) and stromal cell CD163 ${ }^{+}$ TAM proportion (coefficient $=0.76$; 95\% CI: 0.71; 0.8) (Supplementary Table S1). The three NND metrics were highly pairwise correlated, but not as correlated with the count and proportion or with the metrics of $\mathrm{CD} 163^{+}$TAM proximity to cancer cells (Supplementary Figure S1). Meanwhile, the two metrics of CD163 ${ }^{+}$TAM proximity to cancer cells were substantially correlated (coefficient $=0.55$; 95\% CI: 0.49; 0.62) (Supplementary Table S1).

Supplementary Figures S2 and S3 present the associations between all CD163 ${ }^{+}$TAMrelated metrics and clinicopathologic characteristics. Only $p$-values significant after multiple testing adjustment are shown on the plots. Notably, HR-negative (HR-) tumors had significantly higher levels of traditional CD163 ${ }^{+}$TAM count-based metrics and a higher average number of communicating $\mathrm{CD}_{163}{ }^{+}$TAMs as compared patients with HR-positive $(\mathrm{HR}+)$ tumors, but no difference between HR- and HR+ tumors was observed in terms of the NND-based CD163 ${ }^{+}$TAM metrics or the average number of adjacent CD163 ${ }^{+}$. Similarly, higher levels of $\mathrm{CD}_{163^{+}}{ }^{\mathrm{TAM}}$ count-based metrics were associated with higher histologic grade, but no such trend was observed for the NND-based metrics. However, NND-based $\mathrm{CD}_{163}{ }^{+}$TAM metrics were higher in Her2-positive as compared to Her2-negative tumors. 

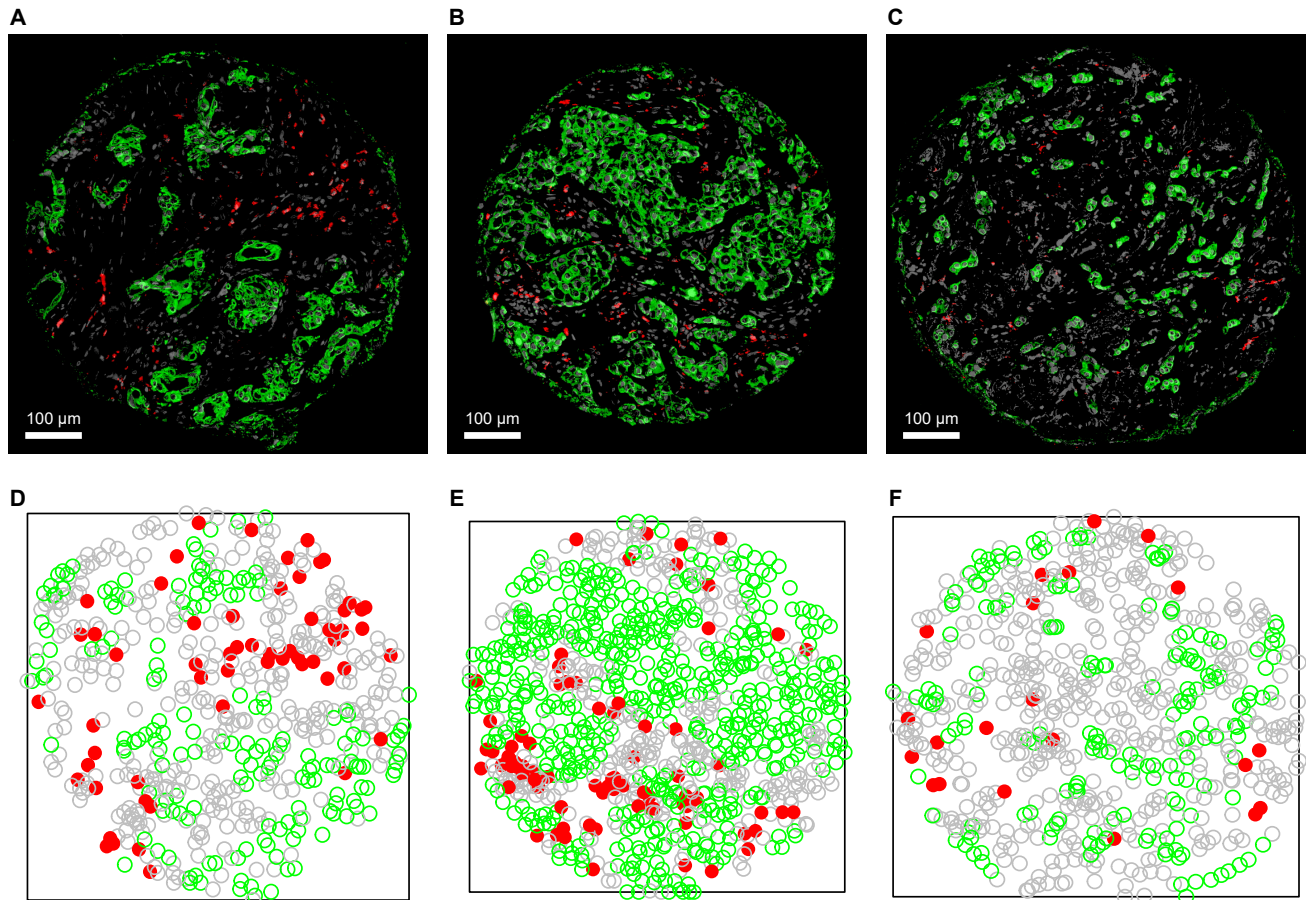

E
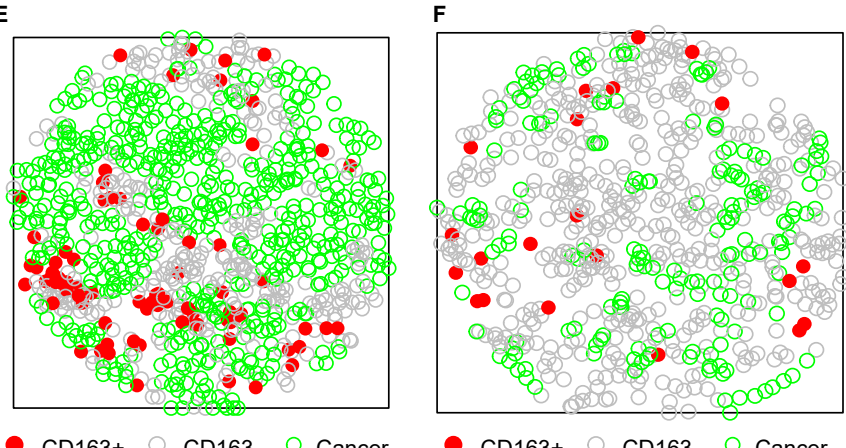

- CD163+ $\bigcirc$ CD163- $\bigcirc$ Cancer

H
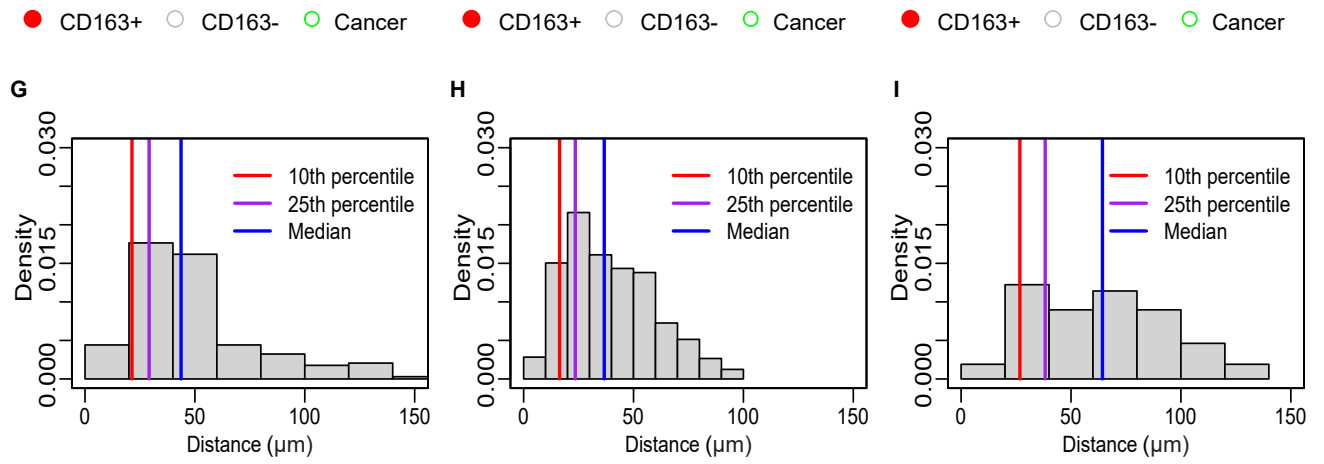

Figure 2. Spatial analysis of CD163 ${ }^{+}$TAMs and cancer cells in breast cancer. IF-IHC images ((A-C); cancer cells green, $\mathrm{CD}_{163^{+}}$TAMs red) with corresponding marked point patterns (D-F) of CD163 ${ }^{+}$ TAMs (red), $C D 163^{-}$cells (grey), and cancer cells (green), and nearest neighbor distance (NND) distributions $(\mathrm{G}-\mathbf{I})$ for cancer cells to the nearest CD163 ${ }^{+}$TAM.

We next explored dichotomization for all $\mathrm{CD}_{163^{+}}$TAM-related metrics. Optimal dichotomization thresholds were identified for each $\mathrm{CD} 163^{+}$TAM-related metric, and all dichotomized count-based and distance-based metrics remained significant predictors of progression-free survival (PFS) after bootstrap-based bias correction and adjustment for multiple testing (Table 2). Table 1 and Supplementary Tables S2-S8 present the associations of all

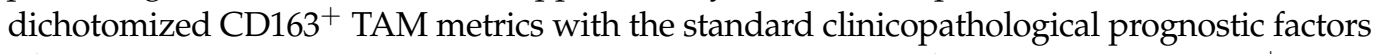
of PFS and adjuvant treatment indicators. Similar to results for continuous CD163 ${ }^{+}$TAM metrics, HR- breast tumors have higher proportions of cases with a high total count of CD163 ${ }^{+}$ TAMs and a high number of adjacent or communicating CD163 ${ }^{+}$TAMs as compared HR+ tumors (Table 1 and Supplementary Tables S6 and S7). Also, high levels of all count-based $\mathrm{CD}_{163}{ }^{+}$TAM metrics were associated with higher histological grade (Table 1 and Supplementary Tables S2, S6 and S7), and high CD163+ TAM NND-based metric were associated with higher proportion of Her2-positive tumors (Supplementary Tables S3-S5). No association with age was observed for any of the CD163+ TAM metrics (Supplementary Table S8). 
Table 2. Bias-adjusted hazard ratios and optimal cutpoints for dichotomized metrics.

\begin{tabular}{|c|c|c|c|c|c|}
\hline Metric & $\begin{array}{l}\text { Dichotomization } \\
\text { Threshold (\#) }\end{array}$ & $\begin{array}{r}\text { Hazard } \\
\text { Ratio (*) }\end{array}$ & LL 95\%CI & UL $95 \%$ CI & $p(\mathcal{E})$ \\
\hline High vs. low number of $\mathrm{CD}^{2} 63^{+}{ }^{\mathrm{T} A M s}$ & $70(12-102)$ & 2.97 & 1.73 & 5.11 & $<0.001$ \\
\hline High vs. low proportion of CD $163^{+}{ }^{\text {TAMs }}$ & $0.038(0.004-0.149)$ & 1.94 & 1.20 & 3.14 & 0.026 \\
\hline High vs. low 10th PCTL of cancer-to-CD163 ${ }^{+}$NNDs & $13(7-55)$ & 0.60 & 0.38 & 0.94 & 0.027 \\
\hline High vs. low 25th PCTL of cancer-to-CD163 ${ }^{+}$NNDs & $17(9-42)$ & 0.46 & 0.26 & 0.82 & 0.026 \\
\hline High vs. low median cancer-to-CD $163^{+}$NND & $19(13-74)$ & 0.48 & 0.27 & 0.85 & 0.026 \\
\hline High vs. low average number of adjacent $\mathrm{CD} 163^{+}$TAMs & $0.034(0.022-0.087)$ & 2.71 & 1.70 & 4.32 & $<0.001$ \\
\hline High vs. low average number of communicating $\mathrm{CD} 163^{+}$TAMs & $46(21-57)$ & 2.96 & 1.80 & 4.87 & $<0.001$ \\
\hline
\end{tabular}

(\#) bootstrap-based 90\% confidence interval in parentheses. (E) adjusted for multiple testing using Holm's method. $\left.{ }^{*}\right)$ bootstrap-based optimistic bias-adjusted hazard ratio.

The highest bias-adjusted effect sizes (hazard ratios) on PFS were observed for the count of $\mathrm{CD} 163^{+}$TAMs per core (HR $\left.=3.0 ; 95 \% \mathrm{CI}: 1.7-5.1 ; p<0.001\right)$ and the number of communicating CD163 ${ }^{+}$TAMs (HR $=3.0 ; 95 \%$ CI: $1.8-4.9 ; p<0.001$ ), followed by the number of $\mathrm{CD} 163^{+}$TAMs adjacent to cancer cells (HR $=2.7 ; 95 \%$ CI: 1.7-4.3; $p<0.001$ ). These $\mathrm{CD} 163^{+}$TAM metrics as well as the median of the cancer cell-to-CD163 ${ }^{+}$TAM NND distribution remained significant predictors of PFS in multivariable Cox models adjusted for significant clinicopathological prognostic factors (Table 3). Each CD163 ${ }^{+}$TAM metric was evaluated in separate multivariable Cox model due to high correlations between these metrics (Supplementary Table S1). The bias-adjusted effect sizes for all CD163 ${ }^{+}$TAM metrics in multivariable Cox models are presented in Supplementary Table S9.

In multivariable Cox models adjusted for significant clinicopathological prognostic factors of PFS, the number of communicating CD163 ${ }^{+}$TAMs had the largest effect size $(\mathrm{HR}=2.3 ; 95 \% \mathrm{CI}: 1.4-4.0 ; p=0.003)$, followed by the median of the CD163+ ${ }^{+} \mathrm{TAM}$ NND distribution (HR $=1.9 ; 95 \% \mathrm{CI}: 1.03-3.4 ; p=0.024)$, the count of $\mathrm{CD}_{163}{ }^{+}$TAMs per core $(\mathrm{HR}=1.9 ; 95 \%$ CI: $1.05-3.4 ; p=0.039)$, and the number of adjacent CD163 ${ }^{+}$TAMs (HR $=1.8$; 95\% CI: $1.1-2.9 ; p=0.024)$. For illustration, Figure 3 shows the Kaplan-Meier survival estimates for these $\mathrm{CD} 163^{+}$TAM metrics dichotomized using the thresholds obtained as the median threshold in 1000 bootstrap samples (Table 2).

The count-based CD163 ${ }^{+}$TAM metrics significant in multivariable Cox models were highly correlated (correlation coefficients ranging from 0.53 to 0.99 , Supplementary Table $\mathrm{S1}$ ), but the median cancer-to-CD163 ${ }^{+}$NND was less correlated with all these count-based $\mathrm{CD}_{163}{ }^{+} \mathrm{TAM}$ metrics (correlation coefficients ranging from -0.12 to -0.33 ; Supplementary Table S1). Thus, for the secondary analysis, we considered combining the strongest countbased predictor (the number of communicating $\mathrm{CD} 163^{+}$TAMs) with the median cancer-to-

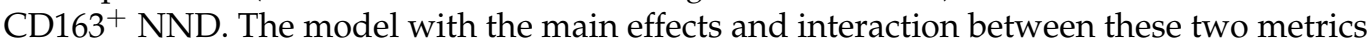
did not yield a significance of interaction term most likely due to the sample size limitation. However, Kaplan-Meier survival estimates of four combinations of low/high number of communicating $\mathrm{CD}_{163}{ }^{+}$TAMs and low/high median cancer-to-CD $163^{+}$NND (Figure 4A) suggested possibly more than 2 different risk categories based on the dichotomized number of communicating $\mathrm{CD}_{163^{+}} \mathrm{TAMs}$ and the median cancer-to-CD163 ${ }^{+} \mathrm{NND}$. Therefore, a combined $\mathrm{CD} 163^{+}$TAM marker was defined with three risk categories: (1) low number of communicating $\mathrm{CD}_{163^{+}}{ }^{\mathrm{TAM}} \mathrm{s}$ and high median cancer-to-CD163 ${ }^{+} \mathrm{NND} ;(2)$ low number of communicating CD163 ${ }^{+}$TAMs and low median cancer-to-CD163 ${ }^{+}$NND; (3) high number of communicating $\mathrm{CD} 163^{+}$TAMs and any median cancer-to-CD163 ${ }^{+}$NND. Figure 4B shows for illustration the corresponding unadjusted Kaplan-Meier survival estimates for the resulting three groups. Table 4 shows the results from the multivariable Cox model including the combined CD163 ${ }^{+}$TAM marker and significant clinicopathological prognostic factors with bootstrap-based optimism correction. This model suggests that with adjustment for known significant clinicopathological prognostic factors, similar extra risk of progression is associated with a high number of communicating $\mathrm{CD} 163^{+} \mathrm{TAMs}(\mathrm{HR}=2.5$; $95 \%$ CI: $1.4-4.9 ; p=0.002)$ and with low median cancer-to-CD163 ${ }^{+} \mathrm{NND}(\mathrm{HR}=2.3 ; 95 \% \mathrm{CI}$ : 
$1.1-4.6 ; p=0.024)$ as compared to the lowest risk group with a low number of communicating $\mathrm{CD}_{163}{ }^{+}$cells and high median cancer-to-CD163 ${ }^{+}$NND (Table 4). The unadjusted KaplanMeier survival estimates for the resulting three groups suggest higher risk associated with a high number of communicating $\mathrm{CD} 163^{+}$TAMs than with how median cancer-to-CD163 ${ }^{+}$ NND because of association between hormone receptor status and combined CD163 ${ }^{+}$TAM marker. Namely, 33/63 (52\%) of tumors with a high number of communicating CD163 ${ }^{+}$ TAMs are HR-, but only 12/81 (15\%) of tumors with a low number of communicating CD163 ${ }^{+}$ TAMs and low median cancer-to-CD163 ${ }^{+}$NND are HR-. Thus, adjustment for hormone receptor status reduces the effect size for a high number of communicating $\mathrm{CD} 163^{+} \mathrm{TAMs}$ in the multivariable Cox model. Meanwhile, the effect of low median cancer-to-CD163 ${ }^{+}$ NND is attributed primarily to HR+ tumors overrepresented in the risk group with low median cancer-to-CD163 ${ }^{+}$NND and a low number of communicating CD163 ${ }^{+}$TAMs. $^{2}$

Table 3. Bias-adjusted results from the parsimonious Cox models with significant CD163 metrics.

\begin{tabular}{|c|c|c|c|c|}
\hline Model with Number of CD163 ${ }^{+}$TAMs & Hazard Ratio (*) & LL 95\% CI & UL 95\% CI & $p$-Value \\
\hline Histological Grade 2 vs. 1 & 2.01 & 0.92 & 4.39 & 0.086 \\
\hline Histological Grade 3 vs. 1 & 2.42 & 1.05 & 5.58 & 0.042 \\
\hline HR- vs. HR+ Hormone Tx compliant & 1.52 & 0.78 & 3.00 & 0.226 \\
\hline HR+ Hormone Tx Non-Compliant vs. Compliant & 2.74 & 1.43 & 5.24 & 0.004 \\
\hline Node Positive vs. Negative & 1.84 & 1.10 & 3.07 & 0.022 \\
\hline Tumor Size $2-5 \mathrm{~cm}$ vs. $<2 \mathrm{~cm}$ & 1.96 & 1.15 & 3.36 & 0.016 \\
\hline Tumor Size $>5 \mathrm{~cm}$ vs. $<2 \mathrm{~cm}$ & 2.93 & 1.53 & 5.61 & 0.002 \\
\hline High vs. Low Number of CD163 ${ }^{+}$TAMs & 1.89 & 1.05 & 3.41 & 0.039 \\
\hline Model with Median Cancer-to-CD163 ${ }^{+}$NND & Hazard Ratio (*) & LL 95\% CI & UL 95\% CI & $p$-Value \\
\hline Histological Grade 2 vs. 1 & 2.35 & 1.08 & 5.11 & 0.036 \\
\hline Histological Grade 3 vs. 1 & 2.87 & 1.26 & 6.53 & 0.015 \\
\hline HR- vs. HR+ Hormone Tx Compliant & 1.90 & 1.00 & 3.61 & 0.055 \\
\hline HR+ Hormone Tx Non-Compliant vs. Compliant & 2.71 & 1.39 & 5.28 & 0.005 \\
\hline Node Positive vs. Negative & 1.78 & 1.06 & 2.97 & 0.032 \\
\hline Tumor Size $2-5 \mathrm{~cm}$ vs. $<2 \mathrm{~cm}$ & 1.85 & 1.08 & 3.17 & 0.029 \\
\hline Tumor Size $>5 \mathrm{~cm}$ vs. $<2 \mathrm{~cm}$ & 2.76 & 1.44 & 5.30 & 0.003 \\
\hline Low vs. High Median Cancer-to-CD163 ${ }^{+}$NND & 1.88 & 1.03 & 3.44 & 0.024 \\
\hline Model with Average Number of Adjacent $C D 163^{+}$TAMs & Hazard Ratio $\left(^{*}\right)$ & LL 95\% CI & UL $95 \%$ CI & $p$-Value \\
\hline Histological Grade 2 vs. 1 & 1.99 & 0.91 & 4.36 & 0.090 \\
\hline Histological Grade 3 vs. 1 & 2.18 & 0.94 & 5.04 & 0.074 \\
\hline HR- vs. HR+ Hormone Tx Compliant & 1.71 & 0.89 & 3.29 & 0.111 \\
\hline HR+ Hormone Tx Non-Compliant vs. Compliant & 2.61 & 1.37 & 4.97 & 0.005 \\
\hline Node Positive vs. Negative & 1.84 & 1.11 & 3.07 & 0.022 \\
\hline Tumor Size $2-5 \mathrm{~cm}$ vs. $<2 \mathrm{~cm}$ & 1.97 & 1.15 & 3.36 & 0.016 \\
\hline Tumor Size $>5 \mathrm{~cm}$ vs. $<2 \mathrm{~cm}$ & 2.76 & 1.44 & 5.28 & 0.003 \\
\hline High vs. Low Average Number of adjacent CD163 ${ }^{+}$TAMs & 1.78 & 1.09 & 2.89 & 0.024 \\
\hline Model with Average Number of Communicating $C D 163^{+}$TAMs & Hazard Ratio $(*)$ & LL 95\% CI & UL $95 \%$ CI & $p$-Value \\
\hline Histological Grade 2 vs. 1 & 2.25 & 1.03 & 4.92 & 0.045 \\
\hline Histological Grade 3 vs. 1 & 2.85 & 1.24 & 6.57 & 0.016 \\
\hline HR- vs. HR+ Hormone Tx Compliant & 1.48 & 0.75 & 2.91 & 0.267 \\
\hline HR+ Hormone Tx Non-Compliant vs. Compliant & 2.55 & 1.32 & 4.92 & 0.008 \\
\hline Node Positive vs. Negative & 1.79 & 1.08 & 2.97 & 0.027 \\
\hline Tumor Size $2-5 \mathrm{~cm}$ vs. $<2 \mathrm{~cm}$ & 1.81 & 1.06 & 3.09 & 0.034 \\
\hline Tumor Size $>5 \mathrm{~cm}$ vs. $<2 \mathrm{~cm}$ & 2.77 & 1.45 & 5.28 & 0.003 \\
\hline High vs. Low Average Number of communicating CD163 ${ }^{+}$TAMs & 2.33 & 1.37 & 3.98 & 0.003 \\
\hline
\end{tabular}

$\left({ }^{*}\right)$ Bootstrap-based optimistic bias-adjusted hazard ratio. 
A

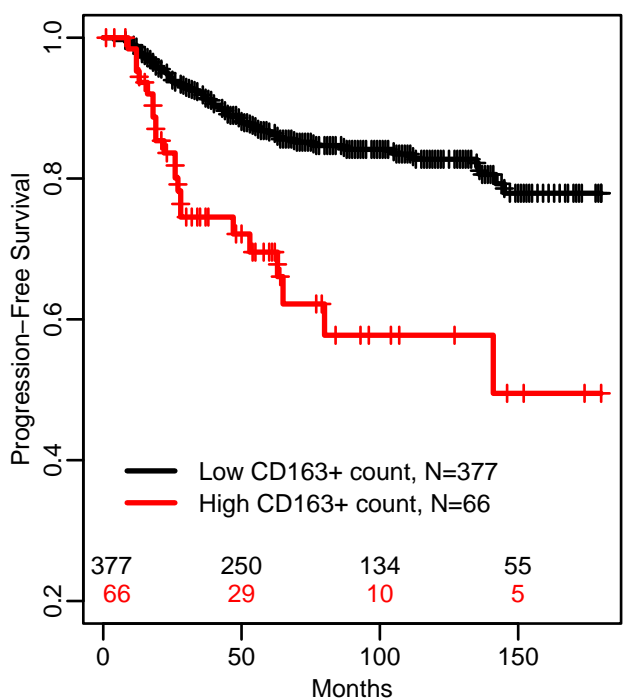

C

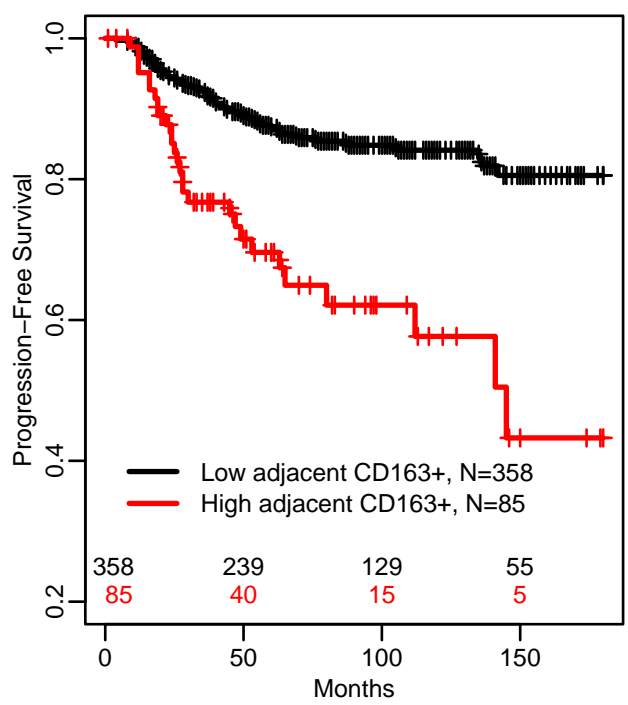

B

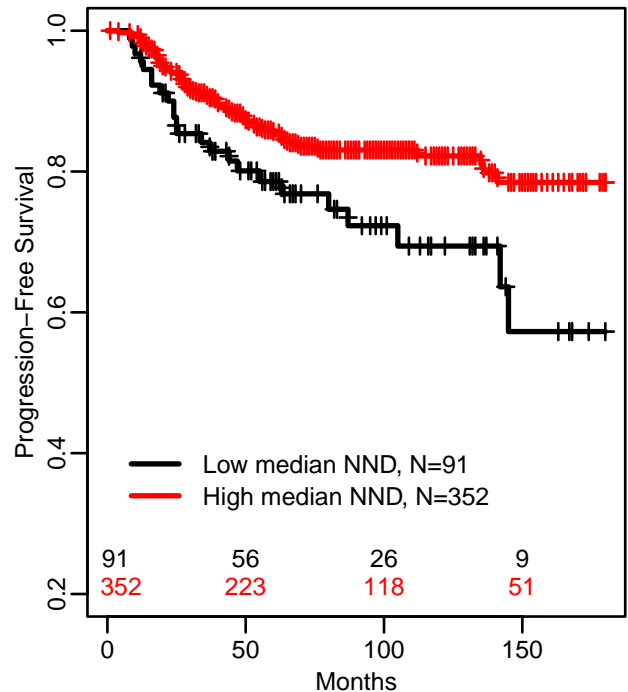

D

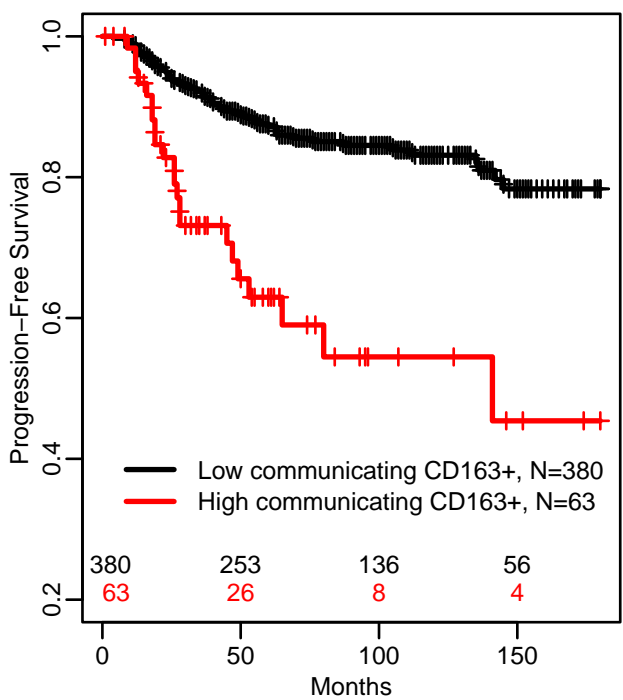

Figure 3. Kaplan-Meier plots of PFS by CD163-related markers with the highest prognostic value in the multivariable Cox model adjusted for significant clinicopathologic risk factors. (A) total number of $\mathrm{CD}_{163}{ }^{+} \mathrm{TAMs}$ in the sampled tumor area (CD163 ${ }^{+}$Count); (B) median nearest neighbor distance (median NND) from cancer cells to CD163 ${ }^{+}$TAMs; (C) the average number of $\mathrm{CD} 163^{+}{ }^{\text {TAMs }}$ within $12 \mu \mathrm{m}$ distance of each cancer cell (adjacent $\mathrm{CD} 163^{+}$); (D) the average number of CD163 ${ }^{+}$TAMs within $250 \mu \mathrm{m}$ distance of each cancer cell (communicating CD163 ${ }^{+}$). 
Table 4. Bias-adjusted results from the parsimonious Cox models with combined CD163 ${ }^{+}$ TAM marker.

\begin{tabular}{|c|c|c|c|c|}
\hline Predictor & Hazard Ratio $\left(^{*}\right)$ & LL 95\% CI & UL $95 \%$ CI & $p$-Value \\
\hline Histological Grade 2 vs. 1 & 2.17 & 0.99 & 4.76 & 0.056 \\
\hline Histological Grade 3 vs. 1 & 2.74 & 1.19 & 6.31 & 0.021 \\
\hline HR- vs. HR+ Hormone Tx Compliant & 1.52 & 0.77 & 3.01 & 0.233 \\
\hline HR+ Hormone Tx Non-Compliant vs. Compliant & 2.67 & 1.35 & 5.28 & 0.007 \\
\hline Node Positive vs. Negative & 1.86 & 1.12 & 3.09 & 0.019 \\
\hline Tumor Size $2-5 \mathrm{~cm}$ vs. $<2 \mathrm{~cm}$ & 1.75 & 1.02 & 3.00 & 0.046 \\
\hline Tumor Size $>5 \mathrm{~cm}$ vs. $<2 \mathrm{~cm}$ & 2.81 & 1.48 & 5.33 & 0.002 \\
\hline $\begin{array}{l}\text { Low vs. High Median Cancer-to-CD163 }{ }^{+} \text {NND } \\
\text { AND Low Average Number of communicating CD163 } \\
+ \text { TAMs }\end{array}$ & 2.29 & 1.14 & 4.63 & 0.024 \\
\hline $\begin{array}{l}\text { High vs. Low Average Number of communicating CD163 } \\
\text { AND High or Low Median Cancer-to-CD163 }{ }^{+} \text {NND }\end{array}$ & 2.47 & 1.42 & 4.29 & 0.002 \\
\hline
\end{tabular}

(*) Bootstrap-based optimistic bias-adjusted hazard ratio.

A

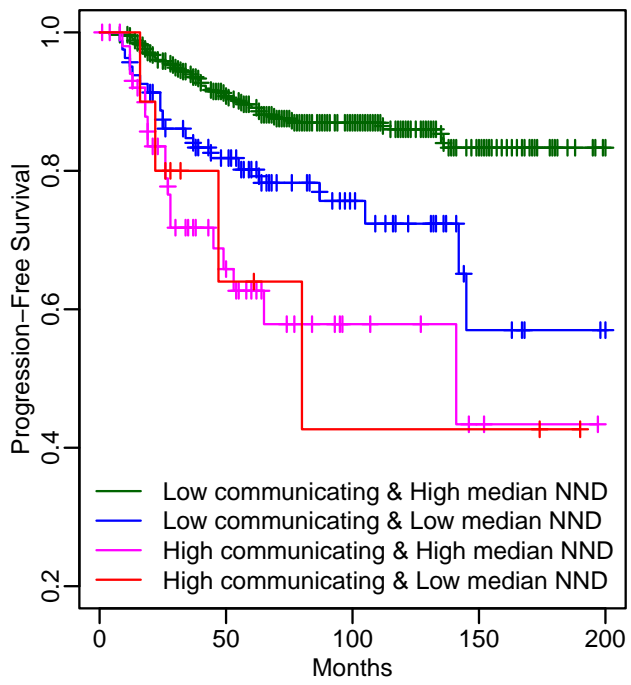

B

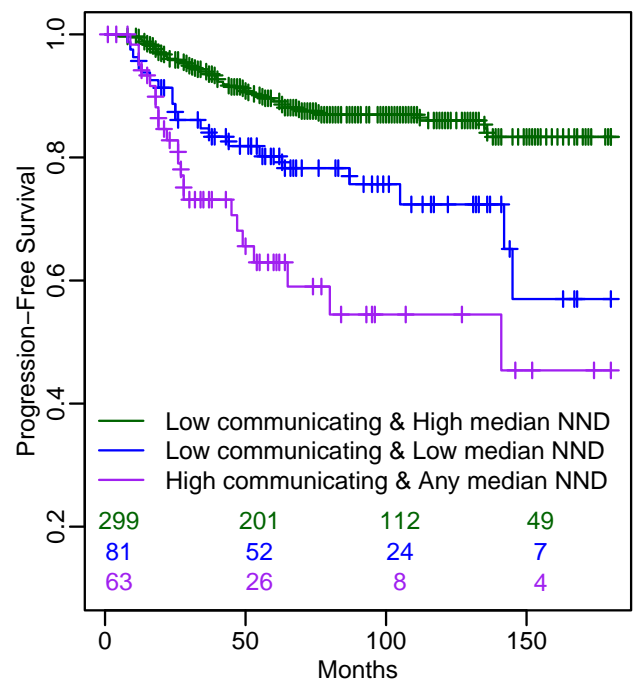

Figure 4. Kaplan-Meier plots of PFS by combined CD163 ${ }^{+}$TAM marker based on high vs. low average number of communicating $\mathrm{CD}_{163^{+}}$cells and high vs. low median NND. (A) by four combinations of low/high average number of communicating $\mathrm{CD}_{163^{+}}$cells and low/high median cancer-to-CD163 ${ }^{+}$ NND; (B) by combined CD163 marker with 3 categories: (1) low average number of communicating $\mathrm{CD} 63^{+}$cells and high median cancer-to-CD163 ${ }^{+} \mathrm{NND} ;(2)$ low average number of communicating $\mathrm{CD}_{163}{ }^{+}$cells and low median cancer-to-CD163 ${ }^{+} \mathrm{NND}$; (3) high average number of communicating $\mathrm{CD}_{163}{ }^{+}$cells and any median cancer-to-CD163 ${ }^{+}$NND.

\section{Discussion}

Mounting evidence indicates that high levels of tumor-associated macrophages (TAMs) $[6,25,36,56]$, and especially CD163 ${ }^{+}$TAMs of the M2-like phenotype [34,37-39], are associated with poor prognosis in breast cancer. However, the published evidence has largely centered on pathologists' visual enumeration of $\mathrm{CD}^{\prime} 63^{+}$TAMs with limited capability to objectively capture prognostic value embedded in the spatial relationship between of $\mathrm{CD}_{163^{+}}$TAMs and cancer cells. In this study, we developed a novel method for objective phenotyping of $\mathrm{CD} 163^{+}$TAMs and present new spatial metrics of proximity of $\mathrm{CD} 163^{+}$TAMs to cancer cells that are independent predictors of clinical outcome in high-risk breast cancer patients treated with adjuvant chemotherapy. The new algorithm identifies $\mathrm{CD}_{163}{ }^{+}$TAMs in an objective manner, independent of subjective assessment by trained pathologists or by subjective thresholding by the operator of the image analysis software. The spatial interactions between cancer cells and CD163 ${ }^{+}$TAMs are quantified 
objectively using the spatial metrics based on nearest neighbor distance (NND) distribution and the average number of $\mathrm{CD}_{163}{ }^{+}$cells either directly in contact with cancer cells (adjacent within $12 \mu \mathrm{m}$ of cancer cells) or within paracrine signaling distance of cancer cells (communicating within $<250 \mu \mathrm{m}$ distance from cancer cells).

Since the circles with $250 \mu \mathrm{m}$ radius are comparable in size with the tissue core with diameter of $600 \mu \mathrm{m}$, the CD163 ${ }^{+}$TAM count and the average count of communicating $\mathrm{CD} 163^{+}$TAMs are highly correlated in our study. However, the average number of communicating $\mathrm{CD}_{163^{+}}$TAMs has the advantage that this metric can be applied directly to histological images of any size (whole tissue, biopsy or TMA) without the need to select a tumor region of interest. Moreover, our results indicate that the average number of communicating $\mathrm{CD} 163^{+}$TAMs is a potentially more informative metric than the traditional density of $\mathrm{CD}_{163^{+}}$TAMs within a sampled area of the tumor. Similarly, all NND-based metrics and the average number of adjacent $\mathrm{CD} 163^{+}$TAMs can be used in tumor images of any size and can also be computed for other immune cell populations and can be applied to other solid tumor types. Although we focused on the analysis of breast cancer data, higher $\mathrm{CD}_{163}{ }^{+}$TAM density has been shown to be correlated with worse prognosis in other cancer subtypes such as cutaneous melanoma [57], colorectal carcinoma [58,59], and ovarian carcinoma [60]. These studies primarily focused on the density of TAMs rather than spatial arrangements of TAMs. However, some studies showed that increased $\mathrm{CD} 63^{+}$TAM prevalence was associated with improved clinical outcomes in colorectal carcinoma [61,62]. Thus, as pointed out by Krijgsman et al. [63], it is critical to characterize the spatial distribution of TAMs in order to fully understand the association between TAMs and clinical outcomes.

Our studies of the spatial distribution of $\mathrm{CD}_{163}{ }^{+}$TAMs differ from previously described quantitative analyses of TAMs in tumor microenvironment in several aspects. First, virtually all reported studies used trained pathologists to analyze tumor specimens, while we developed methodology which allowed for the operator-independent identification of cancer cells and CD163 ${ }^{+}$TAMs in the tumor stroma. Second, we developed a novel method for objective phenotyping of $\mathrm{CD}_{163}{ }^{+}$TAMs that does not rely on subjective identification of $\mathrm{CD} 63^{+}$TAMs. Third, we present spatial metrics that have not been used in other studies of CD163 ${ }^{+}$TAMs in the tumor microenvironment, although NND-based metrics have been investigated for CD8+ T cells in solid tumors in multiple reports.

The role of tumor-infiltrating lymphocytes (TILs) has been an active area of breast cancer research [5-13]. There is a potential for association between metrics based on TILs and TAMs, but such investigation is beyond the scope of this paper, and will be a subject of further research. Another limitation of our study is that we do not consider the association between PD-L1 status and CD163 ${ }^{+}$TAM metrics. Limitations of this study also include the retrospective analysis of archived tissue samples and the use of TMAs, which provide limited sampling of each tumor. For a majority of the patients, only one core was included into the TMA. Our results were internally validated using rigorous statistical analysis, but validation in independent patient cohort(s) and whole-tissue sections will be needed. However, more extensive tumor sampling from whole-tissue sections is expected to provide more accurate data and potentially strengthen the results based on spatial CD163 ${ }^{+}$TAM metrics from limited TMA cores.

\section{Conclusions}

Our results demonstrate for the first time that close spatial proximity of $\mathrm{CD}_{163}{ }^{+} \mathrm{TAMs}$ to cancer cells and the average number of adjacent or communicating CD163 ${ }^{+}$TAMs are independent predictors of unfavorable prognosis in breast cancer. In breast cancer patients who received chemotherapy, we found that the average number of communicating CD163 ${ }^{+}$ TAMs had the largest effect size (hazard ratio) in the multivariable Cox model for PFS adjusted for known significant clinicopathological risk factors and corrected for optimistic bias associated with dichotomization. However, the confidence intervals of this hazard ratio largely overlap with similarly adjusted hazard ratios for the dichotomized median 
cancer-to-CD163 ${ }^{+}$NND or the average number of adjacent CD163 ${ }^{+}$TAMs. Hence, it remains possible that the spatial metrics of median cancer-to-CD163 ${ }^{+} \mathrm{NND}$ or the number of adjacent $\mathrm{CD}_{163}{ }^{+}$TAMs may become more important predictors than the number of communicating $\mathrm{CD}_{163}{ }^{+}$cells in other solid tumors or in distinct breast cancer cohorts, for instance in different breast cancer subtypes or under different treatment.

Methods to identify breast cancer patients with an elevated risk profile based on $\mathrm{CD}_{163}{ }^{+}$TAM-centered tumor markers have the potential to improve clinical management of patients by identifying patients with unfavorable prognosis and opening the way for alternative tumor immune-boosting strategies. The present study builds on past progress of cell density-based tumor analyses and provides new insights into the prognostic significance of spatial proximity of CD163 ${ }^{+}$TAMs to cancer cells using objective and quantitative methodologies. The described automated analysis pipeline is readily applicable to both core needle biopsies and whole-tissue specimens and is expected to be relevant beyond breast cancer. The prognostic values of $\mathrm{CD} 163^{+}$TAM density and spatial proximity to breast cancer cells may also be independent prognostic factors in other solid malignancies.

Supplementary Materials: The following are available online at https:/ / www.mdpi.com/article/ 10.3390/cancers14020308/s1, Figure S1: Scatter plots between all pairs of CD163-related markers. Figure S2: Boxplots of count-based CD163 ${ }^{+}$TAM-related metrics by standard clinicopathologic characteristics., Figure S3: Boxplots of NND-based CD163 ${ }^{+}$TAM-related metrics by standard clinicopathologic characteristics. Table S1: Correlation coefficients with the corresponding $95 \%$ confidence intervals. Table S2: Associations between dichotomized stromal proportion of $\mathrm{CD}_{163}{ }^{+}$cells and other prognostic factors. Table S3: Associations between dichotomized 10th percentile of the NND distribution and other prognostic factors. Table S4: Associations between dichotomized 25th percentile of the NND distribution and other prognostic factors. Table S5: Associations between dichotomized median of the NND distribution and other prognostic factors. Table S6: Associations between the dichotomized number of $\mathrm{CD}_{163^{+}}$cells within $12 \mu \mathrm{m}$ from cancer and other prognostic factors. Table S7: Associations between the dichotomized number of $\mathrm{CD}_{163}{ }^{+}$cells within $250 \mu \mathrm{m}$ from cancer and other prognostic factors. Table S8: Associations between age at diagnosis and dichotomized CD163 markers. Table S9: Bias-adjusted hazard ratios from the multivariate parsimonious Cox models with imputations and including dichotomized CD163 metrics one at a time.

Author Contributions: Conceptualization, H.R. and I.C.; Methodology, H.R., Y.S. and I.C.; Validation, H.R., Y.S. and A.R.P.; Formal Analysis, B.A.M., M.Y., A.R.P. and I.C.; Resources, H.R., C.D.S., J.A.H., A.J.K. and H.H.; Data Curation, Y.S., A.R.P., J.A.H., A.J.K. and H.H.; Writing-Original Draft Preparation, B.A.M., M.Y., H.R. and I.C.; Writing-Review and Editing, B.A.M., M.Y., H.R., I.C., A.R.P., Y.S., J.A.H., A.J.K., C.D.S., H.H., M.T.N., T.T., N.S. and L.L.W.; Visualization, A.R.P. and I.C.; Supervision, H.R. and I.C.; Project Administration, H.R. and I.C.; Funding Acquisition, H.R., C.D.S., J.A.H., A.J.K., H.H., T.T. and I.C. All authors have read and agreed to the published version of the manuscript.

Funding: This study was supported in part by funding from grants from the NCI (R01CA222847 (I.C., H.R.), R01CA164225 (L.L.W.), R01CA223804 (L.L.W.)), DOD (W81XWH1910356 (T.T., I.C.), W81XWH1910357 (H.R.), W81XWH-21-0003 (T.T., H.R., I.C.)), and American Cancer Society (RSG (L.L.W.), MCWCC Institutional Research Grant \#14-247-29 (Y.S.)) and Susan G. Komen Foundation (Promise Award KG091116 (H.R., A.J.K., J.A.H., H.H., C.D.S., A.R.P., I.C.).

Institutional Review Board Statement: The analyses reported in this study have been conducted according to the guidelines of the Declaration of Helsinki and were performed with institutional review board approval of Medical College of Wisconsin (IRB protocol PRO00028590, 02/13/2017).

Informed Consent Statement: Not applicable, the study did not involve human subjects (archival materials only).

Data Availability Statement: The data sets used and/or analyzed during the current study are available from the corresponding authors on request.

Conflicts of Interest: The authors declare no conflict of interest. The funders had no role in the design of the study; in the collection, analyses, or interpretation of data; in the writing of the manuscript, or in the decision to publish the results. 


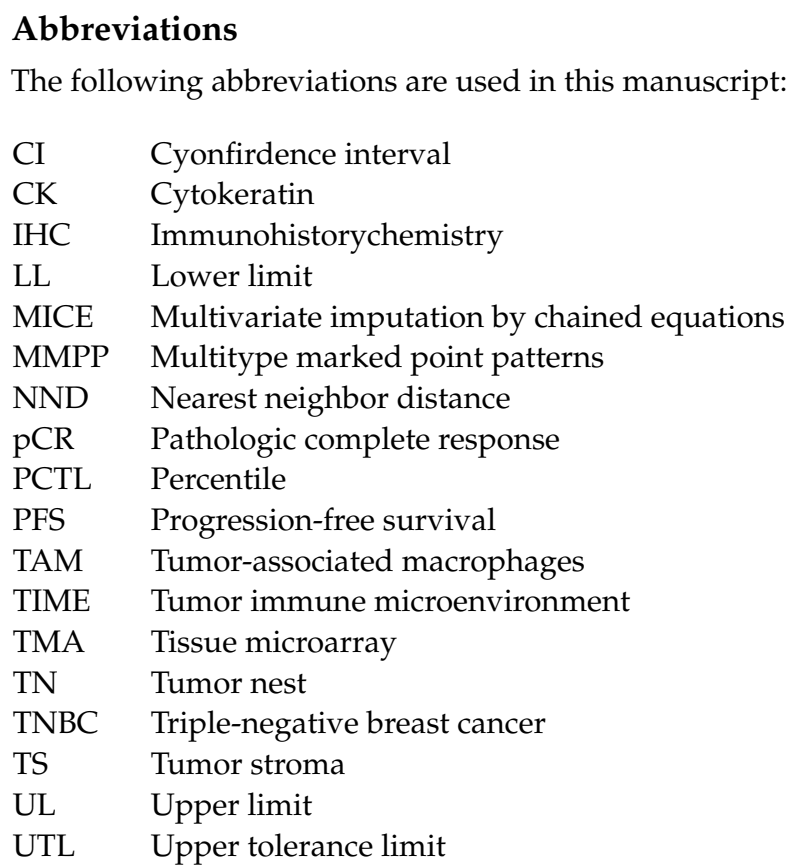

\section{References}

1. Siegel, R.L.; Miller, K.D.; Fuchs, H.E.; Jemal, A. Cancer statistics, 2021. CA Cancer J. Clin. 2021, 71, 7-33. [CrossRef] [PubMed]

2. Opzoomer, J.W.; Sosnowska, D.; Anstee, J.E.; Spicer, J.F.; Arnold, J.N. Cytotoxic Chemotherapy as an Immune Stimulus: A Molecular Perspective on Turning Up the Immunological Heat on Cancer. Front. Immunol. 2019, 10. [CrossRef] [PubMed]

3. Ludwig, K.F.; Du, W.; Sorrelle, N.B.; Wnuk-Lipinska, K.; Topalovski, M.; Toombs, J.E.; Cruz, V.H.; Yabuuchi, S.; Rajeshkumar, N.; Maitra, A.; et al. Small-Molecule Inhibition of Axl Targets Tumor Immune Suppression and Enhances Chemotherapy in Pancreatic Cancer. Cancer Res. 2017, 78, 246-255. [CrossRef] [PubMed]

4. Hu, J.; Zhao, Q.; Kong, L.Y.; Wang, J.; Yan, J.; Xia, X.; Jia, Z.; Heimberger, A.B.; Li, S. Regulation of tumor immune suppression and cancer cell survival by CXCL1/2 elevation in glioblastoma multiforme. Sci. Adv. 2021, 7, eabc2511. [CrossRef] [PubMed]

5. $\quad$ Binnewies, M.; Roberts, E.W.; Kersten, K.; Chan, V.; Fearon, D.F.; Merad, M.; Coussens, L.M.; Gabrilovich, D.I.; Ostrand-Rosenberg, S.; Hedrick, C.C.; et al. Understanding the tumor immune microenvironment (TIME) for effective therapy. Nat. Med. 2018, 24, 541-550. [CrossRef]

6. Fortis, S.P.; Sofopoulos, M.; Sotiriadou, N.N.; Haritos, C.; Vaxevanis, C.K.; Anastasopoulou, E.A.; Janssen, N.; Arnogiannaki, N.; Ardavanis, A.; Pawelec, G.; et al. Differential intratumoral distributions of CD8 and CD163 immune cells as prognostic biomarkers in breast cancer. J. Immunother. Cancer 2017, 5, 1-12.

7. Kim, J.W.; Nam, K.H.; Ahn, S.H.; Park, D.J.; Kim, H.H.; Kim, S.H.; Chang, H.; Lee, J.O.; Kim, Y.J.; Lee, H.S.; et al. Prognostic implications of immunosuppressive protein expression in tumors as well as immune cell infiltration within the tumor microenvironment in gastric cancer. Gastric Cancer 2016, 19, 42-52. [CrossRef]

8. Sato, E.; Olson, S.H.; Ahn, J.; Bundy, B.; Nishikawa, H.; Qian, F.; Jungbluth, A.A.; Frosina, D.; Gnjatic, S.; Ambrosone, C.; et al Intraepithelial CD8+ tumor-infiltrating lymphocytes and a high CD8+/regulatory $\mathrm{T}$ cell ratio are associated with favorable prognosis in ovarian cancer. Proc. Natl. Acad. Sci. USA 2005, 102, 18538-18543. [CrossRef]

9. Nakano, O.; Sato, M.; Naito, Y.; Suzuki, K.; Orikasa, S.; Aizawa, M.; Suzuki, Y.; Shintaku, I.; Nagura, H.; Ohtani, H. Proliferative activity of intratumoral CD8+ T-lymphocytes as a prognostic factor in human renal cell carcinoma: Clinicopathologic demonstration of antitumor immunity. Cancer Res. 2001, 61, 5132-5136.

10. Schumacher, K.; Haensch, W.; Röefzaad, C.; Schlag, P.M. Prognostic significance of activated CD8+ T cell infiltrations within esophageal carcinomas. Cancer Res. 2001, 61, 3932-3936.

11. Matkowski, R.; Gisterek, I.; Halon, A.; Lacko, A.; Szewczyk, K.; Staszek, U.; Pudelko, M.; Szynglarewicz, B.; Szelachowska, J.; Zolnierek, A.; et al. The prognostic role of tumor-infiltrating CD4 and CD8 T lymphocytes in breast cancer. Anticancer. Res. 2009, 29, 2445-2451.

12. Dales, J.P.; Garcia, S.; Carpentier, S.; Andrac, L.; Ramuz, O.; Lavaut, M.N.; Allasia, C.; Bonnier, P.; Charpin, C. Long-term prognostic significance of neoangiogenesis in breast carcinomas: Comparison of Tie-2/Tek, CD105, and CD31 immunocytochemical expression. Hum. Pathol. 2004, 35, 176-183. [CrossRef] [PubMed]

13. Mohamed, S.Y.; Mohammed, H.L.; Ibrahim, H.M.; Mohamed, E.M.; Salah, M. Role of VEGF, CD105, and CD31 in the prognosis of colorectal cancer cases. J. Gastrointest. Cancer 2019, 50, 23-34. [CrossRef] [PubMed]

14. Gao, Z.H.; Li, C.X.; Liu, M.; Jiang, J.Y. Predictive and prognostic role of tumour-infiltrating lymphocytes in breast cancer patients with different molecular subtypes: A meta-analysis. BMC Cancer 2020, 20, 1-14. [CrossRef] [PubMed] 
15. Gong, C.; Anders, R.A.; Zhu, Q.; Taube, J.M.; Green, B.; Cheng, W.; Bartelink, I.H.; Vicini, P.; Wang, B.; Popel, A.S. Quantitative characterization of CD8+ T cell clustering and spatial heterogeneity in solid tumors. Front. Oncol. 2019, 8, 649. [CrossRef]

16. Galon, J.; Costes, A.; Sanchez-Cabo, F.; Kirilovsky, A.; Mlecnik, B.; Lagorce-Pagès, C.; Tosolini, M.; Camus, M.; Berger, A.; Wind, P.; et al. Type, density, and location of immune cells within human colorectal tumors predict clinical outcome. Science 2006, 313, 1960-1964. [CrossRef]

17. Nearchou, I.P.; Gwyther, B.M.; Georgiakakis, E.C.; Gavriel, C.G.; Lillard, K.; Kajiwara, Y.; Ueno, H.; Harrison, D.J.; Caie, P.D. Spatial immune profiling of the colorectal tumor microenvironment predicts good outcome in stage II patients. NPJ Digit. Med. 2020, 3, 1-10. [CrossRef]

18. Castino, G.F.; Cortese, N.; Capretti, G.; Serio, S.; Di Caro, G.; Mineri, R.; Magrini, E.; Grizzi, F.; Cappello, P.; Novelli, F.; et al. Spatial distribution of B cells predicts prognosis in human pancreatic adenocarcinoma. Oncoimmunology 2016, 5, e1085147. [CrossRef]

19. Galon, J.; Mlecnik, B.; Bindea, G.; Angell, H.K.; Berger, A.; Lagorce, C.; Lugli, A.; Zlobec, I.; Hartmann, A.; Bifulco, C.; et al Towards the introduction of the 'Immunoscore' in the classification of malignant tumours. J. Pathol. 2014, 232, 199-209. [CrossRef]

20. Enfield, K.S.; Martin, S.D.; Marshall, E.A.; Kung, S.H.; Gallagher, P.; Milne, K.; Chen, Z.; Nelson, B.H.; Lam, S.; English, J.C.; et al. Hyperspectral cell sociology reveals spatial tumor-immune cell interactions associated with lung cancer recurrence. J. Immunother. Cancer 2019, 7, 1-13. [CrossRef]

21. Barua, S.; Fang, P.; Sharma, A.; Fujimoto, J.; Wistuba, I.; Rao, A.U.; Lin, S.H. Spatial interaction of tumor cells and regulatory T cells correlates with survival in non-small cell lung cancer. Lung Cancer 2018, 117, 73-79. [CrossRef]

22. Nawaz, S.; Heindl, A.; Koelble, K.; Yuan, Y. Beyond immune density: Critical role of spatial heterogeneity in estrogen receptornegative breast cancer. Mod. Pathol. 2015, 28, 766-777. [CrossRef]

23. Yuan, Y. Modelling the spatial heterogeneity and molecular correlates of lymphocytic infiltration in triple-negative breast cancer. J. R. Soc. Interface 2015, 12, 20141153. [CrossRef]

24. Mani, N.L.; Schalper, K.A.; Hatzis, C.; Saglam, O.; Tavassoli, F.; Butler, M.; Chagpar, A.B.; Pusztai, L.; Rimm, D.L. Quantitative assessment of the spatial heterogeneity of tumor-infiltrating lymphocytes in breast cancer. Breast Cancer Res. 2016, 18, 1-10. [CrossRef] [PubMed]

25. Egelston, C.A.; Avalos, C.; Tu, T.Y.; Rosario, A.; Wang, R.; Solomon, S.; Srinivasan, G.; Nelson, M.S.; Huang, Y.; Lim, M.H.; et al. Resident memory CD8+ T cells within cancer islands mediate survival in breast cancer patients. JCI Insight 2019, 4, e130000. [CrossRef] [PubMed]

26. Li, X.; Gruosso, T.; Zuo, D.; Omeroglu, A.; Meterissian, S.; Guiot, M.C.; Salazar, A.; Park, M.; Levine, H. Infiltration of CD8+ T cells into tumor cell clusters in triple-negative breast cancer. Proc. Natl. Acad. Sci. USA 2019, 116, 3678-3687. [CrossRef] [PubMed]

27. Pelekanou, V.; Villarroel-Espindola, F.; Schalper, K.A.; Pusztai, L.; Rimm, D.L. CD68, CD163, and matrix metalloproteinase 9 (MMP-9) co-localization in breast tumor microenvironment predicts survival differently in ER-positive and-negative cancers. Breast Cancer Res. 2018, 20, 49. [CrossRef] [PubMed]

28. Baxevanis, C.N.; Sofopoulos, M.; Fortis, S.P.; Perez, S.A. The role of immune infiltrates as prognostic biomarkers in patients with breast cancer. Cancer Immunol. Immunother. 2019, 68, 1671-1680. [CrossRef] [PubMed]

29. Gordon, S.; Martinez, F.O. Alternative activation of macrophages: Mechanism and functions. Immunity 2010, 32, 593-604. [CrossRef] [PubMed]

30. Spranger, S. Mechanisms of tumor escape in the context of the T-cell-inflamed and the non-T-cell-inflamed tumor microenvironment. Int. Immunol. 2016, 28, 383-391. [CrossRef] [PubMed]

31. Mills, C.D.; Kincaid, K.; Alt, J.M.; Heilman, M.J.; Hill, A.M. M-1/M-2 macrophages and the Th1/Th2 paradigm. J. Immunol. 2000, 164, 6166-6173. [CrossRef]

32. Yang, J.; Li, X.; Liu, X.; Liu, Y. The role of tumor-associated macrophages in breast carcinoma invasion and metastasis. Int. J. Clin. Exp. Pathol. 2015, 8, 6656.

33. Wu, K.; Lin, K.; Li, X.; Yuan, X.; Xu, P.; Ni, P.; Xu, D. Redefining tumor-associated macrophage subpopulations and functions in the tumor microenvironment. Front. Immunol. 2020, 11, 1731. [CrossRef]

34. Lau, S.K.; Chu, P.G.; Weiss, L.M. CD163A specific marker of macrophages in paraffin-embedded tissue samples. Am. J. Clin. Pathol. 2004, 122, 794-801. [CrossRef]

35. Tang, X. Tumor-associated macrophages as potential diagnostic and prognostic biomarkers in breast cancer. Cancer Lett. 2013, 332, 3-10. [CrossRef]

36. Medrek, C.; Pontén, F.; Jirström, K.; Leandersson, K. The presence of tumor associated macrophages in tumor stroma as a prognostic marker for breast cancer patients. BMC Cancer 2012, 12, 306. [CrossRef]

37. Jeong, H.; Hwang, I.; Kang, S.H.; Shin, H.C.; Kwon, S.Y. Tumor-associated macrophages as potential prognostic biomarkers of invasive breast cancer. J. Breast Cancer 2019, 22, 38-51. [CrossRef]

38. Jamiyan, T.; Kuroda, H.; Yamaguchi, R.; Abe, A.; Hayashi, M. CD68-and CD163-positive tumor-associated macrophages in triple negative cancer of the breast. Virchows Archiv 2020, 477, 767-775. [CrossRef] [PubMed]

39. Klingen, T.A.; Chen, Y.; Aas, H.; Wik, E.; Akslen, L.A. Tumor-associated macrophages are strongly related to vascular invasion, non-luminal subtypes, and interval breast cancer. Hum. Pathol. 2017, 69, 72-80. [CrossRef] [PubMed] 
40. Tiainen, S.; Tumelius, R.; Rilla, K.; Hämäläinen, K.; Tammi, M.; Tammi, R.; Kosma, V.M.; Oikari, S.; Auvinen, P. High numbers of macrophages, especially M2-like (CD 163-positive), correlate with hyaluronan accumulation and poor outcome in breast cancer. Histopathology 2015, 66, 873-883. [CrossRef] [PubMed]

41. Yang, M.; Li, Z.; Ren, M.; Li, S.; Zhang, L.; Zhang, X.; Liu, F. Stromal infiltration of tumor-associated macrophages conferring poor prognosis of patients with basal-like breast carcinoma. J. Cancer 2018, 9, 2308. [CrossRef] [PubMed]

42. Peck, A.R.; Witkiewicz, A.K.; Liu, C.; Klimowicz, A.C.; Stringer, G.A.; Pequignot, E.; Freydin, B.; Yang, N.; Ertel, A.; Tran, T.H.; et al. Low levels of Stat5a protein in breast cancer are associated with tumor progression and unfavorable clinical outcomes. Breast Cancer Res. 2012, 14, R130. [CrossRef]

43. Peck, A.R.; Witkiewicz, A.K.; Liu, C.; Stringer, G.A.; Klimowicz, A.C.; Pequignot, E.; Freydin, B.; Tran, T.H.; Yang, N.; Rosenberg, A.L.; et al. Loss of nuclear localized and tyrosine phosphorylated Stat5 in breast cancer predicts poor clinical outcome and increased risk of antiestrogen therapy failure. J. Clin. Oncol. 2011, 29, 2448. [CrossRef]

44. Sato, T.; Tran, T.H.; Peck, A.R.; Liu, C.; Ertel, A.; Lin, J.; Neilson, L.M.; Rui, H. Global profiling of prolactin-modulated transcripts in breast cancer in vivo. Mol. Cancer 2013, 12, 59. [CrossRef]

45. Hahn, G.J.; Meeker, W.Q. Statistical Intervals: A Guide for Practitioners; John Wiley \& Sons: Hoboken, NJ, USA, 2011 ; Volume 92.

46. Van, R.G.; Drake, F. Python 3 Reference Manual; CreateSpace: Scotts Valley, CA, USA, 2009.

47. Stoyan, D.; Stoyan, H. Fractals, Random Shapes and Point Fields: Methods of Geometrical Statistics; Wiley-Blackwell: Hoboken, NJ, USA, 1994; Volume 302

48. Francis, K.; Palsson, B.O. Effective intercellular communication distances are determined by the relative time constants for cyto/chemokine secretion and diffusion. Proc. Natl. Acad. Sci. USA 1997, 94, 12258-12262. [CrossRef]

49. Baddeley, A.; Turner, R. Spatstat: An R package for analyzing spatial point patterns. J. Stat. Softw. 2005, 12, 1-42. [CrossRef]

50. Therneau, T.M.; Atkinson, E.J. An Introduction to Recursive Partitioning Using the RPART Routines; Technical Report; Technical Report Mayo Foundation; Mayo Clinic: Rochester, MN, USA, 1997.

51. Holm, S. A simple sequentially rejective multiple test procedure. Scand. J. Stat. 1979, 6, 65-70.

52. Steyerberg, E.W. Clinical Prediction Models; Springer: Berlin/Heidelberg, Germany, 2019.

53. Van Buuren, S.; Groothuis-Oudshoorn, K. mice: Multivariate imputation by chained equations in R. J. Stat. Softw. 2011, 45, 1-67. [CrossRef]

54. Rubin, D.B. Multiple Imputation for Nonresponse in Surveys; John Wiley \& Sons: Hoboken, NJ, USA, 2004 ; Volume 81.

55. RC Team. R: A Language and Environment for Statistical Computing; R Foundation for Statistical Computing: Vienna, Austria, 2018.

56. Thompson, E.; Taube, J.M.; Elwood, H.; Sharma, R.; Meeker, A.; Warzecha, H.N.; Argani, P.; Cimino-Mathews, A.; Emens, L.A. The immune microenvironment of breast ductal carcinoma in situ. Mod. Pathol. 2016, 29, 249-258. [CrossRef]

57. Salmi, S.; Siiskonen, H.; Sironen, R.; Tyynelä-Korhonen, K.; Hirschovits-Gerz, B.; Valkonen, M.; Auvinen, P.; Pasonen-Seppänen, S. The number and localization of CD68+ and CD163+ macrophages in different stages of cutaneous melanoma. Melanoma Res. 2019, 29, 237. [CrossRef]

58. Cui, Y.L.; Li, H.K.; Zhou, H.Y.; Zhang, T.; Li, Q. Correlations of tumor-associated macrophage subtypes with liver metastases of colorectal cancer. Asian Pac. J. Cancer Prev. 2013, 14, 1003-1007. [CrossRef]

59. Yang, C.; Wei, C.; Wang, S.; Shi, D.; Zhang, C.; Lin, X.; Dou, R.; Xiong, B. Elevated CD163+/CD68+ ratio at tumor invasive front is closely associated with aggressive phenotype and poor prognosis in colorectal cancer. Int. J. Biol. Sci. 2019, 15, 984. [CrossRef]

60. Etzerodt, A.; Moulin, M.; Doktor, T.K.; Delfini, M.; Mossadegh-Keller, N.; Bajenoff, M.; Sieweke, M.H.; Moestrup, S.K.; AuphanAnezin, N.; Lawrence, T. Tissue-resident macrophages in omentum promote metastatic spread of ovarian cancer. J. Exp. Med. 2020, 217. [CrossRef] [PubMed]

61. Koelzer, V.H.; Canonica, K.; Dawson, H.; Sokol, L.; Karamitopoulou-Diamantis, E.; Lugli, A.; Zlobec, I. Phenotyping of tumorassociated macrophages in colorectal cancer: Impact on single cell invasion (tumor budding) and clinicopathological outcome. Oncoimmunology 2016, 5, e1106677. [CrossRef] [PubMed]

62. Cavnar, M.J.; Turcotte, S.; Katz, S.C.; Kuk, D.; Gönen, M.; Shia, J.; Allen, P.J.; Balachandran, V.P.; D’ Angelica, M.I.; Kingham, T.P.; others. Tumor-associated macrophage infiltration in colorectal cancer liver metastases is associated with better outcome. Ann. Surg. Oncol. 2017, 24, 1835-1842. [CrossRef] [PubMed]

63. Krijgsman, D.; De Vries, N.L.; Andersen, M.N.; Skovbo, A.; Tollenaar, R.A.; Møller, H.J.; Hokland, M.; Kuppen, P.J. CD163 as a biomarker in colorectal cancer: The expression on circulating monocytes and tumor-associated macrophages, and the soluble form in the blood. Int. J. Mol. Sci. 2020, 21, 5925. [CrossRef] [PubMed] 